\title{
Mechanisms preventing trench defect formation in InGaN/GaN quantum well structures using hydrogen during GaN barrier growth
}

\author{
Fabien Massabuau*, Menno Kappers, Colin Humphreys, Rachel Oliver. \\ Department of Materials Science and Metallurgy, University of Cambridge, 27 Charles Babbage Road, Cambridge CB3 0FS, United \\ Kingdom
}

Received XXXX, revised XXXX, accepted XXXX

Published online XXXX

Key words: III-nitrides, trench defect, epitaxy, hydrogen.

${ }^{*}$ Corresponding author: e-mail fm350@cam.ac.uk, Phone: +44 1223 334044, Fax: +44 1223334437

Here we study the mechanisms underlying a method used to limit the formation of trench defects in In$\mathrm{GaN} / \mathrm{GaN}$ quantum well structures by using $\mathrm{H}_{2}$ in the carrier gas for the growth of $\mathrm{GaN}$ barriers. The method leads to a complete removal of the trench defects by preventing the formation of basal plane stacking faults from which trench defects originate, as well as preventing the formation of stacking mismatch boundaries. The penalty paid for the absence of trench defects is the formation of InGaN wells with gross well- width fluctuations where the $\mathrm{H}_{2}$ gas has etched away the indium locally.

Were a fully formed trench defect (stacking mismatch boundary opened as $\mathrm{V}$-shaped ditch) already exists in the structure, the $\mathrm{GaN}$ barrier growth method using $\mathrm{H}_{2}$ results in a strongly disturbed structure of the quantum well stack in the enclosed region, with the quantum wells and barriers being in places significantly thinner than their counterparts in the surrounding material.

Copyright line will be provided by the publisher

1 Introduction Owing to a tuneable bandgap spanning the visible and ultraviolet spectral range, III-Nitrides achieve a plethora of applications including blue and green light emitting diodes (LEDs) [1], laser diodes [2], and solar cells [3]. However III-Nitride materials are afflicted with a very high density of defects - point defects, threading dislocations, trench defects, etc. - which deteriorate the device performance [4-7]. The trench defect is a flaw which is commonly found in InGaN quantum wells (QWs). It consists of a basal-plane stacking fault (BSF) located in the QW stack and bounded by a vertical stacking mismatch boundary (SMB) which opens up at the sample surface into a trench enclosing a material with altered emission properties [8]. In LEDs, where a p-cap is required, it has been demonstrated that trench defects have a significant negative impact on the structure and efficiency of the devices [7]. Additionally, trench defects are more likely to form in green-emitting samples, which suggests that they may play a role in the decrease in efficiency observed on samples emitting at longer wavelength, commonly known as the "green gap" problem $[1,9]$. It is therefore important to be able to suppress the formation of trench defects in an InGaN QW structure.

In the past, several studies have attempted to prevent or reduce their occurrence by, for example, applying a $\mathrm{H}_{2}$ treatment during $\mathrm{GaN}$ barrier growth $[10,11]$ or InGaN growth [12], using AlGaN as barrier [13], or modifying the $\mathrm{GaN}$ growth temperature [10,14-16], the InGaN growth temperature [17] the InGaN growth rate [18], the trimethylindium flux [17], the pressure [19], or the substrate misorientation [20]. However these studies assessed only the resulting surface topography and did not investigate the structural impact of the various growth methods on trench defects. Here we will assess the use of $\mathrm{H}_{2}$ as carrier 
gas during GaN barrier growth as a trench defect reduction technique, with an emphasis on the (sub-surface) structural effects of the method, and hence identify elements of the mechanisms by which $\mathrm{H}_{2}$ prevents trench formation.

2 Methods Three blue-emitting 10-period InGaN/GaN QW structures were grown by metal organic vapour phase epitaxy using a Thomas Swan $6 \times 2$ in. closecoupled showerhead reactor. Trimethylgallium (TMG), trimethylindium (TMI) and ammonia $\left(\mathrm{NH}_{3}\right)$ were used as precursors with hydrogen $\left(\mathrm{H}_{2}\right)$ and nitrogen $\left(\mathrm{N}_{2}\right)$ as carrier gases. GaN pseudo-substrates, consisting of $c a .5$ $\mu \mathrm{m}$ of $\mathrm{GaN}$ grown on $c$-plane sapphire with a miscut of $0.25 \pm 0.10^{\circ}$ towards $(11 \overline{2} 0)$ at $1020^{\circ} \mathrm{C}$ following deposition of a $30 \mathrm{~nm}$-thick $\mathrm{GaN}$ nucleation layer at $540^{\circ} \mathrm{C}$, were employed. The InGaN QWs were grown at a constant temperature of $759^{\circ} \mathrm{C}$ with a TMG flow of $1.5 \mathrm{sccm}$ (or $4.4 \mu \mathrm{mol}_{\mathrm{min}}{ }^{-1}$ ) and a rather high TMI flow of 300 sccm (or $24.2 \mu \mathrm{mol} . \mathrm{min}^{-1}$ ) in order to deliberately favour the formation of trench defects [17]. For the GaN barrier, a protective $c a .1 \mathrm{~nm}$ thick layer was first deposited at the same temperature and same TMG flow as for the QWs. Then the temperature was ramped to $860^{\circ} \mathrm{C}$ over $90 \mathrm{~s}$, during which an additional $1 \mathrm{~nm}$ of material was grown. Finally the growth of the barrier proceeded at high temperature and TMG flow of $20 \mathrm{sccm}$ (or $67.2 \mu \mathrm{mol}_{\mathrm{min}}{ }^{-1}$ ) for $36 \mathrm{~s}$, leading to an overall barrier thickness of $\mathrm{ca} .7$ $\mathrm{nm}$. The three samples were grown using different carrier gas mixtures for the growth of the GaN barriers (See Figure 1). The GaN barriers in sample "All $\mathrm{N}_{2}$ " were grown using solely $10 \mathrm{slm}$ of $\mathrm{N}_{2}$. Another sample, referred to as "All_ $\mathrm{H}_{2} / \mathrm{N}_{2}$ ", utilised a mixture of $\mathrm{N}_{2}$ and $\mathrm{H}_{2}$ for the growth of the GaN barriers; as illustrated in Figure 1 the $\mathrm{H}_{2} / \mathrm{N}_{2}$ flux was increased from $0 / 10$ slm to $7 / 3$ slm during the temperature ramp-up, and maintained at $7 / 3$ slm during the growth of $\mathrm{GaN}$ barrier at high temperature. Finally a third sample, called "Half $\mathrm{H}_{2} / \mathrm{N}_{2}$ ", was designed with the first $5 \mathrm{GaN}$ barriers similar to those in All $\mathrm{N}_{2}$ and the last 5 barriers similar to those in sample All $\mathrm{H}_{2} / \mathrm{N}_{2}$ in order to assess the impact of $\mathrm{H}_{2}$ during barrier growth on trench defects which are already present in the structure.

$\mathrm{X}$-ray diffraction (XRD) was employed to characterise the thickness and composition of the active region of the samples using $\omega$ - $2 \theta$-scan around the GaN 002 reflection [21]. The results, summarised in Table 1, show that the $\mathrm{GaN}$ barriers are found to be thicker when $\mathrm{H}_{2} / \mathrm{N}_{2}$ was em-

Table 1 QW composition (x) and thickness $\left(\mathrm{t}_{I n G a N}, \mathrm{t}_{\text {GaN }}\right)$ determined by XRD for the samples investigated here.

\begin{tabular}{|c|c|c|c|c|}
\hline Sample & No. QWs & $\begin{array}{c}\mathrm{t}_{\text {InGaN }} \\
(\mathrm{nm})\end{array}$ & $\begin{array}{c}x \\
(\%)\end{array}$ & $\begin{array}{r}\mathrm{t}_{\text {GaN }} \\
(\mathrm{nm})\end{array}$ \\
\hline All_N $N_{2}$ & $10\left(\mathrm{~N}_{2}\right)$ & $3.3 \pm 0.1$ & $16 \pm 1$ & $6.6 \pm 0.1$ \\
\hline All $H_{2} / \mathrm{N}_{2}$ & $10\left(\mathrm{H}_{2} / \mathrm{N}_{2}\right)$ & $3.1 \pm 0.1$ & $11 \pm 1$ & $7 \pm 0.1$ \\
\hline \multirow{2}{*}{ Half_H $\mathrm{H}_{2} / \mathrm{N}_{2}$} & $5\left(\mathrm{~N}_{2}\right)$ & $3.3 \pm 0.1$ & $16 \pm 1$ & $6.6 \pm 0.1$ \\
\hline & $5\left(\mathrm{H}_{2} / \mathrm{N}_{2}\right)$ & $3.1 \pm 0.1$ & $11 \pm 1$ & $7.8 \pm 0.1$ \\
\hline
\end{tabular}
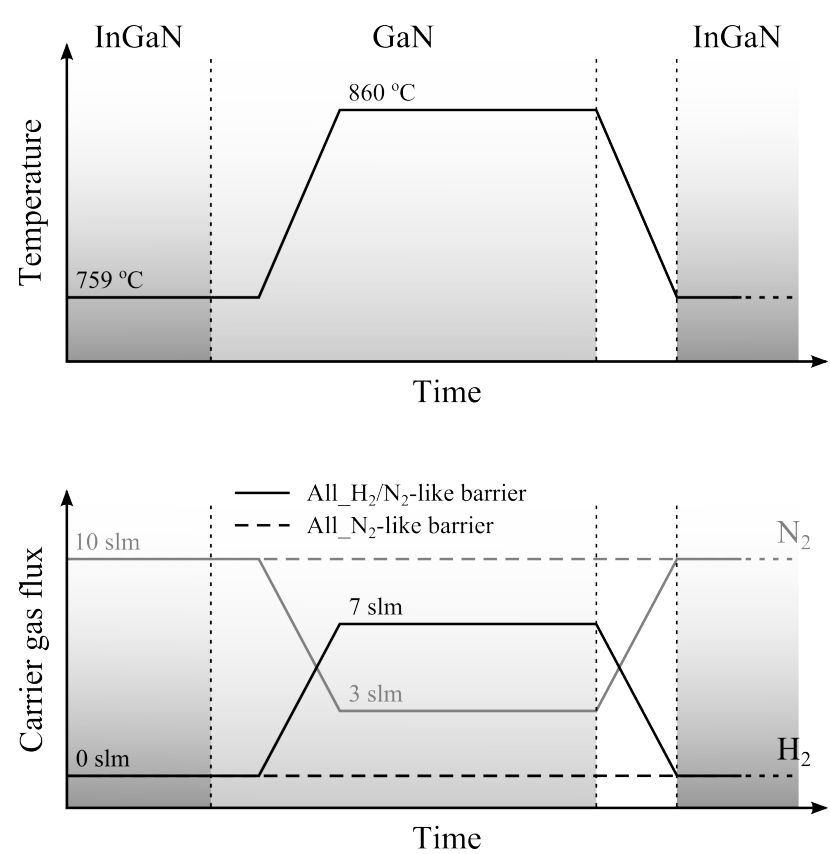

Figure 1 Schematic showing the evolution of temperature and carrier gas flux with time during $\mathrm{GaN}$ barrier growth when $\mathrm{H}_{2} / \mathrm{N}_{2}$ is used (full line) or only $\mathrm{N}_{2}$ (dotted line).

ployed instead of $\mathrm{N}_{2}$, in line with reports that $\mathrm{H}_{2}$ increases the growth rate of $\mathrm{GaN}$ [22]. Additionally we note that the QWs followed by a barrier grown using $\mathrm{H}_{2} / \mathrm{N}_{2}$ are thinner on average ( $3.1 \pm 0.1 \mathrm{~nm}$ as opposed to $3.3 \pm 0.1 \mathrm{~nm})$ and contain less indium $(11 \pm 1 \%$ as compared to $16 \pm 1 \%)$ than their counterpart followed by $\mathrm{GaN}$ using $\mathrm{N}_{2}$. This observation can be explained by the fact that, as we will see later, gross well width fluctuations (GWWFs) [23] are present in the samples grown under $\mathrm{H}_{2} / \mathrm{N}_{2}$, as shown in Ref. [24], thus leading to seemingly thinner QWs of lower composition as measured by XRD (because the thickness and composition reported by XRD are an average of the QW thickness and composition in regions with and without GWWFs). Photoluminescence measurements showed that all three samples exhibit the same peak emission wavelength of $461 \pm 1 \mathrm{~nm}$, suggesting that the main effect of the use of $\mathrm{H}_{2} / \mathrm{N}_{2}$ is to deplete the QW locally (creating GWWFs), but do not result in a global reduction of the QW composition.

The topography of the samples was obtained by atomic force microscopy (AFM) using a Veeco Dimension 3100 operating in tapping mode.

The sub-surface structure of the samples was obtained by transmission electron microscopy (TEM). The presence of BSFs was investigated using diffraction-contrast imaging on a JEOL $4000 \mathrm{EX}$ operating at $400 \mathrm{kV}$, whilst the QW morphology was observed under Z-contrast imaging by high-angle annular dark field scanning TEM (HAADFSTEM) in an FEI Tecnai Osiris operating at $200 \mathrm{kV}$. 

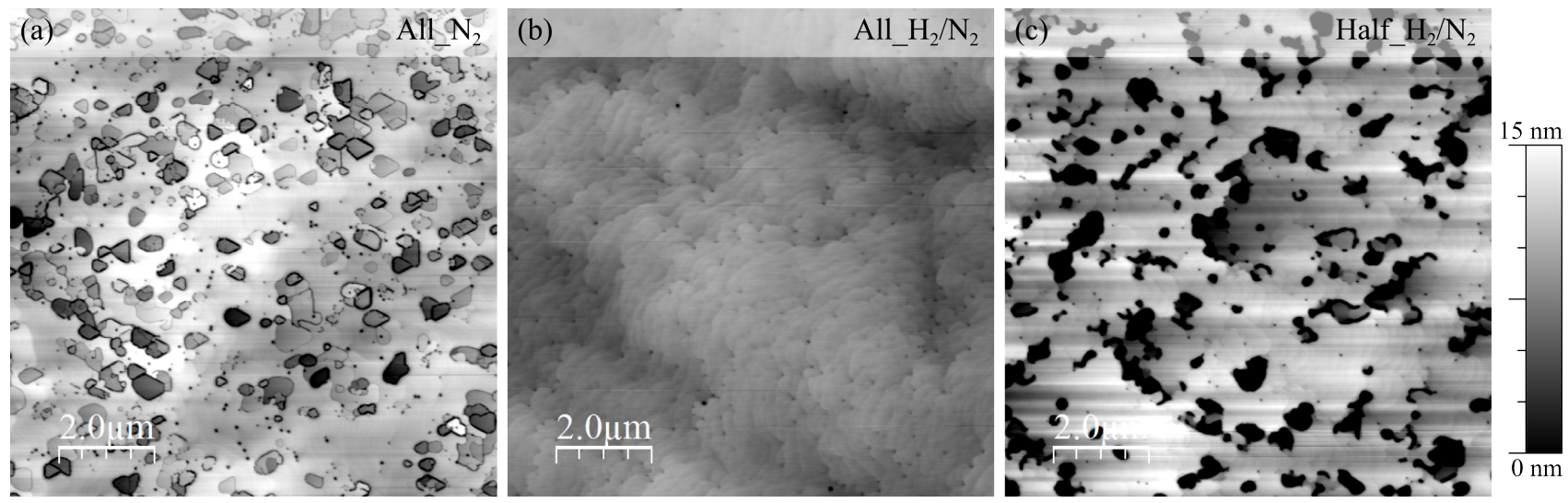

Figure $210 \mu m \times 10 \mu m$ AFM scans of samples All_N $\mathrm{N}_{2}$ (a), All_ $\mathrm{H}_{2} / \mathrm{N}_{2}$ (b), and Half_ $\mathrm{H}_{2} / \mathrm{N}_{2}$ (c). To highlight the variations in prominence of the trench defects across the three samples, a common Z-scale of $15 \mathrm{~nm}$ is employed.

\section{Results and discussions}

3.1 Impact of $\mathrm{H}_{2}$ on trench defect formation The surface morphology of the samples is shown in Figure 2. In line with previous reports [10,11], it is shown clearly in Figure 2(a)-(b) that the use of $\mathrm{H}_{2}$ during $\mathrm{GaN}$ barrier growth leads to a strong reduction of the trench defect density, with the density dropping from $4 \times 10^{8} \mathrm{~cm}^{-2}$ to below $2 \times 10^{5} \mathrm{~cm}^{-2}$ between sample All $\mathrm{N}_{2}$ and All $\mathrm{H}_{2} / \mathrm{N}_{2}$. However, it remains unclear whether $\mathrm{H}_{2}$ entirely prevents the formation of a trench defect - through preventing BSFs from forming in the active region - or if the BSF and SMB actually have formed but this is not reflected on the surface morphology. In the latter case, the defects may still be affecting the optical properties of the materials despite not being visible at the surface by AFM. The sub-surface structure of the samples thus was analysed to ascertain either hypothesis.

The samples have been investigated in cross-section by TEM (not shown here), exciting the $\mathbf{g}=(1 \overline{1} 00)$ diffraction conditions in order to highlight the BSFs in the structures - hence highlighting the presence of trench defects [8]. Whilst BSFs could be observed in sample All_N $\mathrm{N}_{2}$, no BSF could be found in sample All_H $\mathrm{H}_{2} / \mathrm{N}_{2}$. (It can also be noted that all the QWs in sample All_H $\mathrm{H}_{2} / \mathrm{N}_{2}$ exhibit GWWFs [23] despite the growth of a protective low temperature $\mathrm{GaN}$ capping layer and the fact that $\mathrm{H}_{2}$ is used only during the growth of $\mathrm{GaN}$ at high temperature, in agreement with report from $\mathrm{Hu}$ et al. [24].) This confirms that $\mathrm{H}_{2}$ impairs the formation of trench defects by preventing the formation of BSFs, or by eliminating them. We formulate two conjectures to explain this: (1) $\mathrm{H}_{2}$ affects the morphology of the GaN layer which in turn helps relieve the strain in the following QW [22,25] hence making the formation of a BSF less favourable; or (2) since the growth of GaN under $\mathrm{H}_{2}$ generates GWWFs in the QW located below it, the InGaN QW is etched preferentially at the BSF location, where the strain state and perhaps the composition of the $\mathrm{QW}$ is different. It is possible that the low temperature $\mathrm{GaN}$ capping layer does not fully cover the InGaN QW, resulting in the QW being etched preferentially in these regions during barrier growth under $\mathrm{H}_{2}$ (Hu et al. indeed showed that growing a thicker GaN capping layer would prevent the formation of GWWFs [24]). Nevertheless, the experiments described thus far do not reveal if trench defects are suppressed by $\mathrm{H}_{2}$ through the elimination of BSFs or by preventing their formation.

3.2 Impact of $\mathrm{H}_{2}$ on existing trench defects In order to evaluate the impact of $\mathrm{H}_{2}$ on existing trench defects, sample Half_H $\mathrm{H}_{2} / \mathrm{N}_{2}$ was grown. Based on our previous results the design of the sample was such that the lower 5 QW repeats were grown using $\mathrm{N}_{2}$ to act as the trench defect generation part of the structure, followed by the growth of $5 \mathrm{QW}$ repeats grown using $\mathrm{H}_{2} / \mathrm{N}_{2}$.

Figure 3 shows an HAADF-STEM image of the QW stack of sample Half_H $\mathrm{H}_{2} / \mathrm{N}_{2}$ in a region non affected by trench defects. In line with our previous observations, it can be clearly seen that the growth of the lower $5 \mathrm{QW}$ repeats using only $\mathrm{N}_{2}$ results in QWs with uniform thickness (akin to sample All $\mathrm{N}_{2}$ ), while the growth of the upper

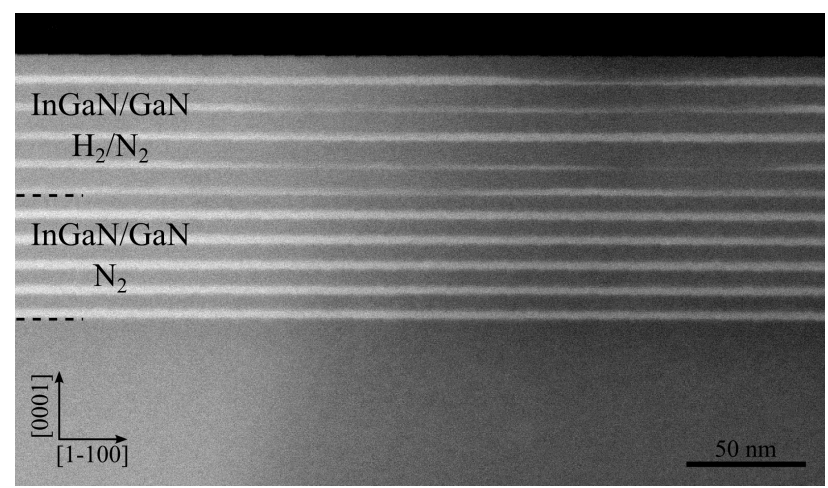

Figure 3 HAADF-STEM image of the QW stack in sample Half_H $\mathrm{H}_{2} / \mathrm{N}_{2}$, observed along the $\langle 11 \overline{2} 0\rangle$ zone-axis. 

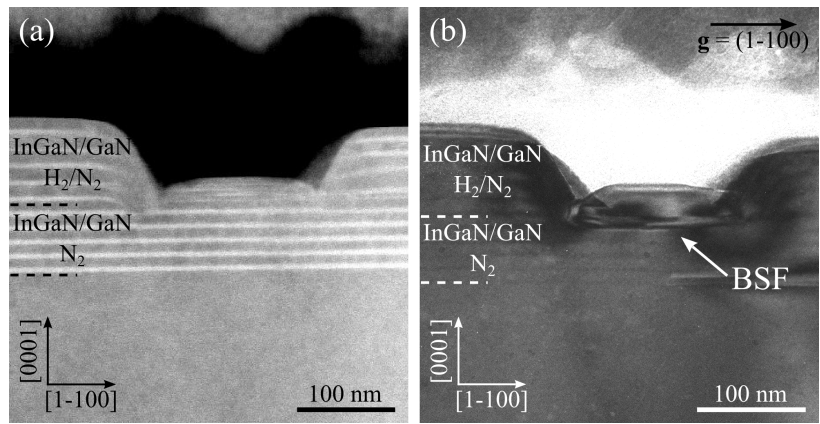

Figure 4 (a) HAADF-STEM image of a typical trench defect in sample Half_ $\mathrm{H}_{2} / \mathrm{N}_{2}$ highlighting the highly disturbed QW stack in the enclosed area, observed along the $\langle 11 \overline{2} 0\rangle$ zone-axis. (b) Bright field TEM image of the same region, using $\mathbf{g}=(1 \overline{1} 00)$ showing the presence of a BSF at the bottom of the trench defects.

5 QW repeats using $\mathrm{H}_{2} / \mathrm{N}_{2}$ results in QWs with GWWFs (akin to sample All_ $\mathrm{H}_{2} / \mathrm{N}_{2}$ ).

AFM of sample Half_H $\mathrm{H}_{2} / \mathrm{N}_{2}$ (See Figure 2(c)) shows a trench defects density of $2 \times 10^{8} \mathrm{~cm}^{-2}$, which is intermediate between that of sample All_N $\mathrm{N}_{2}$ and sample $\mathrm{All} \_\mathrm{H}_{2} / \mathrm{N}_{2}$, as expected since only a stack of 5 QWs instead of 10 is contributing to the formation of trench defects [26]. However, as can be clearly seen in Figure 2 from our use of a common Z-scale, the difference in height between the enclosed region in trench defects and the surrounding material (i.e. the prominence) of sample $\mathrm{Half}_{-} \mathrm{H}_{2} / \mathrm{N}_{2}$ is significantly different than that of sample All $\mathrm{N}_{2}$ or of any trench defects grown in similar samples $[17,27]$. On this sample we recorded prominences as low as $-40 \mathrm{~nm}$ (i.e. the enclosed region is $40 \mathrm{~nm}$ below the surrounding material) while in $10 \mathrm{QW}$ samples similar to All_ $\mathrm{N}_{2}$ the prominence was rarely lower than $-5 \mathrm{~nm}$.

The sub-surface structure of trench defects in sample Half $\mathrm{H}_{2} / \mathrm{N}_{2}$ was therefore investigated by cross-sectional TEM. It is worth mentioning that, as expected from the sample design, all the trench defects originated from the bottom half of the QW stack, where $\mathrm{N}_{2}$ only was utilised. Figure 4 shows a typical trench defect observed by Zcontrast HAADF-STEM and diffraction-contrast TEM. The presence of BSF located in the first half of the QW stack is evidenced by diffraction-contrast TEM using $\mathbf{g}=(1 \overline{1} 00)$, while HAADF-STEM highlights the structure of the QW stack enclosed between the V-shaped ditch. It can be seen that the trench defect is lowered-centred, with a strong negative prominence of approximately $-45 \mathrm{~nm}$. The InGaN QWs and GaN barriers in the enclosed region of the defect are significantly thinner compared to that in the surrounding material, resulting in the strongly negative prominence of the defect. This indicates that $\mathrm{H}_{2}$ has a strong effect on the growth rate of the material grown in the enclosed region (both $\mathrm{GaN}$ and $\mathrm{InGaN}$ ). In a previous study we attributed the prominence of trench defects to the presence of the $\mathrm{V}$-shaped ditch acting as a "negative
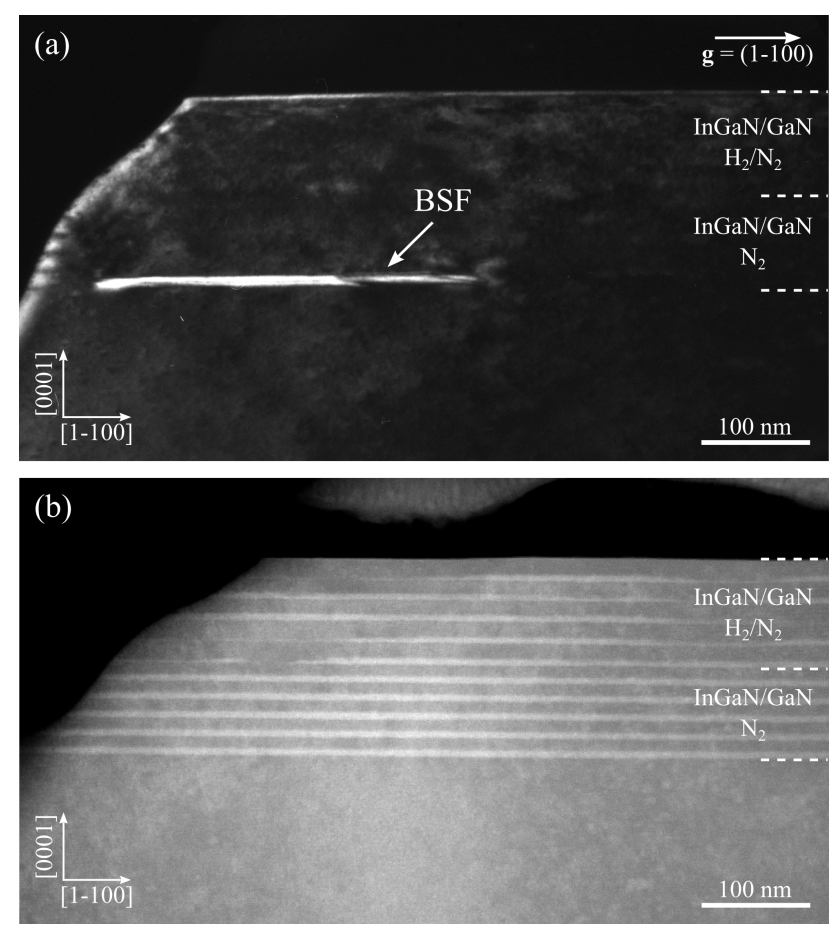

Figure 5 (a) HAADF-STEM image of a typical trench defect in sample Half_H $\mathrm{H}_{2} / \mathrm{N}_{2}$ highlighting the highly disturbed QW stack in the enclosed area, observed along the $\langle 11 \overline{2} 0\rangle$ zone-axis. (b) Bright field TEM image of the same region, using $\mathbf{g}=(1 \overline{1} 00)$ showing the presence of a BSF at the bottom of the trench defects.

mask" for selective area epitaxy [27]. However it was still unclear why some defects were lowered-centred, some level-centred, and some raised-centred [8]. We assumed that a complex interplay of growth conditions, and composition as well as strain in the material may account for the growth of the (partially relaxed) enclosed region of the trench defects. The use of $\mathrm{H}_{2}$ seems to complicate the mechanisms at play even further. More work to understand the structural and optical properties of the enclosed region of trench defects is ongoing.

In many occurrences, incomplete trench defects were noticed, whereby BSFs could be observed in the QW stack but not connected to any SMB or V-shaped pit (See Figure 5). Given that the BSFs are expected to be bounded by a $\mathrm{SMB}$, the presence of such incomplete trench defects indicates that the SMBs, akin to the BSFs, are removed when $\mathrm{H}_{2}$ is used during $\mathrm{GaN}$ growth. Interestingly we note in Figure 5 that the QW stack grown above the BSF is similar to the stack with no BSF under it, and irrespectively of whether $\mathrm{N}_{2}$ or $\mathrm{H}_{2} / \mathrm{N}_{2}$ is employed. This contrasts strongly with the observations made earlier on "complete" trench defects where the part of the stack grown using $\mathrm{H}_{2} / \mathrm{N}_{2}$ was highly disturbed. This suggests that the V-shaped ditch is the cause for such behaviour, which corroborates the suggestion that the growth of the enclosed region is driven by selective area epitaxy mechanisms. 
4 Conclusion In conclusion, we investigated the impact of using a mixture of $\mathrm{H}_{2}$ and $\mathrm{N}_{2}$ as carrier gas for the growth of $\mathrm{GaN}$ barriers as a means to limit the formation of trench defects. We highlighted that this method leads to a complete removal of the trench defects, but at the expense of forming GWWFs. We found that the mechanism by which trench defects are removed is by preventing the formation of BSFs and SMBs. In the case where existing trench defects (SMB opened as V-shaped ditch) are overgrown with $\mathrm{QWs}$ and $\mathrm{GaN}$ barriers using $\mathrm{H}_{2}$ carrier gas, this treatment leads to a strongly disturbed structure of the QW stack in the enclosed region, as well as an overall disturbed surface morphology.

Acknowledgements This work has been funded in part by the EPSRC (under EP/H0495331).

\section{References}

[1] S. Nakamura, M. Senoh, N. Iwasa, and S. I. Nagahama, Japanese Journal of Applied Physics 34, 797 (1995).

[2] S. Nakamura, M. Senoh, S. Nagahama, N. Iwasa, T. Yamada, T. Matsushita, H. Kiyoku, and Y. Sugimoto, Japanese Journal of Applied Physics 35, 74 (1996).

[3] O. Jani, I. Ferguson, C. Honsberg, and S. Kurtz, Applied Physics Letters 91, 132117 (2007).

[4] T. Sugahara, H. Sato, M. Hao, Y. Naoi, S. Kurai, and S. Tottori, Japanese Journal of Applied Physics 37(4), 398 (1998).

[5] A. Armstrong, T. Henry, D. Koleske, M. Crawford, K. Westlake, and S. Lee, Applied Physics Letters 101(16), $162102(2012)$

[6] S. Chichibu, A. Uedono, T. Onuma, T. Sota, B. Haskell, S. Denbaars, J. Speck, and S. Nakamura, Applied Physics Letters 86, 021914 (2005).

[7] F. C. P. Massabuau, M. J. Davies, F. Oehler, S. Pamenter, E. Thrush, M. Kappers, A. Kovács, T. Williams, M. Hopkins, C. Humphreys, P. Dawson, R. Dunin-Borkowski, J. Etheridge, D. Allsopp, and R. Oliver, Applied Physics Letters 105(11), 112110 (2014).

[8] F. P. Massabuau, S. L. Sahonta, L. Trinh-Xuan, S. Rhode, T. Puchtler, M. Kappers, C. Humphreys, and R. Oliver, Applied Physics Letters 101, 212107 (2012).

[9] M. Krames, O. Shchekin, R. Mueller-Mach, G. Mueller, L. Zhou, G. Harbers, and M. Craford, Journal of Display Technology 3(2), 160 (2007).

[10] S. Ting, J. Ramer, D. Florescu, V. Merai, B. Albert, A. Parekh, D. Lee, D. Lu, D. Christini, L. Liu, and E. Armour, Journal of Applied Physics 94(3), 1461 (2003).

[11] D. Florescu, S. Ting, J. Ramer, D. Lee, V. Merai, A. Parkeh, D. Lu, E. Armour, and L. Chernyak, Applied Physics Letters 83(1), 33 (2003).

[12] E. Taylor, F. Fang, F. Oehler, P. Edwards, M. Kappers, K. Lorenz, E. Alves, C. McAleese, C. Humphreys, and R. Martin, Semiconductor Science and Technology 28(6), 065011 (2013).

[13] D. Iida, S. Lu, S. Hirahara, K. Niwa, S. Kamiyama, and K. Ohkawa, Journal of Crystal Growth 448, 105 (2016).
[14] M. Senthil Kumar, J. Park, Y. Lee, S. Chung, C. H. Hong, and E. K. Suh, Journal of Physics D: Applied Physics 40(17), 5050 (2007).

[15] M. Senthil Kumar, Y. Lee, J. Park, S. Chung, C. H. Hong, and E. K. Suh, Materials Chemistry and Physics 113(1), 192 (2009).

[16] J. Smalc-Koziorowska, E. Grzanka, R. Czernecki, D. Schiavon, and M. Leszczyski, Applied Physics Letters 106(10), 101905 (2015).

[17] F. P. Massabuau, A. Le Fol, S. Pamenter, F. Oehler, M. Kappers, C. Humphreys, and R. Oliver, Physica Status Solidi (a) 211(4), 740-743 (2014).

[18] T. Hikosaka, T. Shioda, Y. Harada, K. Tachibana, N. Sugiyama, and S. Nunoue, Phys. Status Solidi C 8(78), 2016 (2011).

[19] T. Suzuki, M. Kaga, K. Naniwae, T. Kitano, K. Hirano, T. Takeuchi, S. Kamiyama, M. Iwaya, and I. Akasaki, Japanese Journal of Applied Physics 52, 08JB27 (2013).

[20] F. P. Massabuau, C. Tartan, R. Traynier, W. Blenkhorn, M. Kappers, P. Dawson, C. Humphreys, and R. Oliver, Journal of Crystal Growth 386, 88 (2014).

[21] M. Vickers, M. Kappers, T. Smeeton, E. Thrush, J. Barnard, and C. Humphreys, Journal of Applied Physics 94(3), 1565 (2003).

[22] R. Czernecki, E. Grzanka, J. Smalc-Koziorowska, S. Grzanka, D. Schiavon, G. Targowski, J. Plesiewicz, P. Prystawko, T. Suski, P. Perlin, and M. Leszczynski, Journal of Crystal Growth 414, 38 (2015).

[23] N. Vander Laak, R. Oliver, M. Kappers, and C. Humphreys, Applied Physics Letters 90(12), 121911 (2007).

[24] Y. L. Hu, R. Farrell, C. Neufeld, M. Iza, S. Cruz, N. Pfaff, D. Simeonov, S. Keller, S. Nakamura, S. DenBaars, U. Mishra, and J. Speck, Applied Physics Letters 100(16), 161101 (2012).

[25] P. Ren, N. Zhang, B. Xue, Z. Liu, J. Wang, and J. Li, Journal of Physics D: Applied Physics 49(17), 175101 (2016).

[26] S. L. Sahonta, M. Kappers, D. Zhu, T. Puchtler, T. Zhu, S. Bennett, C. Humphreys, and R. Oliver, Phys. Status Solidi A 210, 195 (2013).

[27] F. P. Massabuau, L. Trinh-Xuan, D. Lodie, E. Thrush, D. Zhu, F. Oehler, T. Zhu, M. Kappers, C. Humphreys, and R. Oliver, Journal of Applied Physics 113(7), 073505 (2013). 